\title{
SARCOPTIDES PLUMICOLES DES OISEAUX DU MAROC
}

\author{
Par Jean GAUD et M.-L. PETITOT
}

L'étude des sarcoptidés plumicoles ou Analgesidær est à peu près délaissée depuis les travaux, déjà anciens, de Trouessart. L'examen de ces acariens ectoparasites mériterait d'ètre repris. A. Bonnet et J. Timon David exprimaient cette opinion dans un mémoire paru ici même (1). Ils envisageaient l'intérêt de leưr répartition biogéographique, les singularités certaines de leur cycle évolutif (P. Mégnin, 1874), leur rôle inocılateur probable chez les oiseaux.

Cette dernière suggestion avait éveillé notre intérêt. Nous avons espéré, en particulier, trouver parmi les analgésidés les hôtes intermédiaires de quelques-uns des nombreux icestodes aviaires dont le cycle est encore inconnu. Dans cet esprit nous avons examiné, tant au Maroc qu'en Indochine, un grand nombre de ces acariens recueillis sur divers oiseaux. Cette recherche, disons-le tout cie suite, a été négative. Les formes larvaires de cestodes sont extrêmement rares si, toutefois, elles se rencontrent, à l'intérieur des sarcoptidés plumicoles. Sur plus d'un millier d'analgésidés, recutillis sur des oiseaux porteurs de cestodes et examinés avec soin, nous avons seulement trouvé deux images pouvant, à la rigueur, évoquer la présence d'un organisme enkysté. La nature exacte de ces inclusions n'a pu être précisée.

Ce résultat négatif, non concluant, ne mériterait pas publication. Mais, au cours de nos recherches, nous avons acquis quelque expérience sur la récolte et la manipulation de ces petits acariens. Cette expérience peut être utile à un chercheur désireux de poursuivre des études sur les parasites plumicoles.

Par ailleurs, la distribution des différentes espèces d'analgésidés parmi les groupements aviaires pose d'intéressants problèmes concernant la spécificité parasitaire, ainsi que la phylétique ornithologique elle-même (M. André, 1938).

Nous croyons intéressant, à ce titre, de signaler les espèces

(1) Ces Annales, x11, 1934, 257.

Ans, de Parasitologie, T. XXIII, Nos $1-2,-1948$, p. 35-46. 
d'analgésidés rencontrés au Maroc, avec indication des hôtes chez qui ils ont été recueillis.

\section{Techniques}

A. Récolte. - Le plus fréquemment, en ce qui concerne les oiseaux sauvages, c'est sur l'animal mort que l'on doit rechercher les analgésidés. Plusieurs cas peuvent se présenter.

1. Oiseau íué fraîchement. - A moins que l'on ne désire avoir les parasites plumicoles vivants, le meilleur et le seul moyen d'obtenir toute la faune d'un oiseau est de le dépouiller immédiatement et d'immerger la peau dans un bocal fermé rempli d'alcool à $70^{\circ}$. $\mathrm{Au}$ bout de 24 heures au moins de séjour, on agite fortement et longtemps le bocal et on vide l'alcool dans un verre conique. Les parasites plumicoles se rassemblent assez vite au fond. Quelques mouvements de rotation imprimés au verre facilitent cette sédimentation.

Lorsque les oiseaux sont tués par des pourvoyeurs lointains, sur les compétences ornithologiques desquels le doute est permis, il peut être plus commode de se faire envoyer les peaux sèches dans un récipient fermant parfaitement. L'identification de l'oiseau est plus facile. Un lavage à l'alcool à $70^{\circ}$ des parois du récipient ayant servi à l'envoi permet de récupérer les parasites ayant quitté la peau.

Ce procédé de " détrempe " des peaux immédiatement isolées est le seul qui permette d'obtenir les parasites prédateurs peu abondants sur l'oiseau (Dermanyssus, larves d'lxodidés et de Thrombididés).

Si l'on désire éviter la mise en peau, on pourra recueillir un assez grand nombre des parasites en sectionnant seulement les deux ailes et en opérant sur elles. Les rémiges contiennent, en effet, la majeure partie des analgésidés.

Si on désire recueillir les parasites vivants, il faut faire les lavages de la peau, des ailes ou des bocaux à l'eau. Il est alors presque indispensable d'abaisser la tension superficielle de celle-ci (par l'addition de taurocholate de soude p. ex.). Faute de cette précaution, les sarcoptidés ne sont pas mouillés. Un grand nombre demeure sur les parois. Ceux qui sont entraînés flottent sur la surface de l'eau, ce qui rend difficile leur concentration.

2. Oiseau mort depuis quelque temps. - Inutile de rechercher les analgésidés chez un oiseau mort depuis longtemps et aban- 
donné dans la nature. Ces petits acariens ont déjà été dévorés par divers insectes et acariens prédateurs ou détriticoles (fourmis, ploques, tyroglyphes, glyciphages, syringophiles, etc...). Ce sont ces prédateurs qui seront trouvés dans les plumes des oiseaux abandonnés trop longtemps et non les vrais parasites.

Si l'oiseau est mort depuis quelques heures, deux à douze ou même plus, mais a été protégé contre les prédateurs, les sarcoptidés plumicoles se sont rassemblés dans les plumes de la tête et du cou (1). Inutile done de détacher les ailes. Si on désire éviter la mise en peau, le mieux sera de prélever les plumes de la tête et du cou et d'en garnir un tube de verre de taille appropriée en les tassant modérément. Le tube sera bouché avec un bouchon de liège peu serré ou, mieux, avec un capuchon de soie à bluter bien ajusté. Au bout de 24 à 48 heures, tous les analgésidés vivants se concentrent dans les plumes superficielles. Ils paraissent rester vivants très longtemps, à la température du laboratoire, dans ces conditions ; un mois, parfois deux mois, si l'humidité atmosphérique est forte. Beaucoup de nymphes ont ainsi le temps d'évoluer en acariens adultes (2). Lorsqu'on veut prélever les analgésidés ainsi concentrés, prendre à la pince les plumes dont le duvet inférieur porte les acariens et les agiter dans un liquide (alcool à $70^{\circ}$ ou eau " mouillante $")$.

3. Oiseaux vivants. - Le meilleur moyen, encore très imparfait, est de poudrer avec un insecticide (D.D.T., H.C.H.) les plumes de l'oiseau et de mettre celui-ci dans une cage, plancher plein retiré, placée au-dessus d'un plateau garni d'alcool à $70^{\circ}$. On recueille les parasites morts dans cet alcool au bout de 24 heures.

$\mathrm{Si}$ on désire recueillir des parasites vivants, couper les rémiges ou les arracher (3) et les laver à l'eau « mouillante ».

B. Manipulations. - Pour repêcher les analgésidés dans le bain qui a servi à les réunir, le mieux est d'opérer avec un compte-gouttes très effilé sous contrôle d'une loupe binoculaire. Le liquide contenant les acariens sera versé dans un grand verre de montre. Un

(1) Dans le cas d'oiseaux d’espèces différentes ayant demeuré quelques heares en contact étroit (dans une gibecière par exemple), il est souvent impossible d'affirmer que tel acarien provient de tel hôte.

(2) L'elevage serantí peut-être realisable dans ces conditions. Le difficile est d'être sûr que les plumes nouvelles données pour l'alimentation ne contiennent pas elles-mêmes des acariens.

(3) Cette dernière solution, qui paraît barbare, est souvent préférable pour l'oiseau. Les rémiges repoussent aussitôt après arrachement. Après section, au contraire, l'oiseau reste incapable de voler pendant des mois. 
mouvement de balancement circulaire, rapide et de faible amplitude, imprimé à ce verre de montre, ramènera très rapidement tous les acariens en son centre.

Pour garder les analgésidés une fois triés, on peut utiliser des tronçons de verre à pipette de $8 \mathrm{~cm}$. de long, fermés à leur extrémité inférieure par un diaphragme de soie à bluter. Les acariens sont " crachés " dans ces petits tubes par le compte-gouttes qui a servi à les trier. S'il s'agit d'animaux morts, le ou les tubes destinés à les recueillir seront immergés dans l'alcool à $70^{\circ}$ garnissant une conserve de Borel sur les deux tiers de sa hauteur. L'étiquette, s'il y a lieu, sera roulée et introduite dans la partie supérieure du petit tube. Pour un transport éventuel, un petit bouchon empêche les fuites ou les mélanges d'acariens.

S'il s'agit d'animaux vivants, le petit tube sera immergé dans de l'eau additionnée d'un produit mouillant pendant le temps du triage. En fin d'opération. le petit tube est retiré. Un séchage sommaire est pratiqué en apposant le diaphragme de soie à bluter contre un papier buvard. Le tube est alors bouché.

Pour un élevage éventuel, des tubes de ce principe mais d'un calibre plus fort pourraient être utilisés.

Ces petits tubes sont très pratiques pour transporter les acariens de liquide en liquide, pour les déshydratations par exemple. Le montage au baume donne en effet les préparations les plus jolies et les plus solides. Aucun éclaircissement préalable n'est nécessaire. Si l'on désire se dispenser des manipulations requises par le montage au baume, la gomme au chloral de Faure peut aussi être employée. La gomme de Berlèse éclaircit trop les petites espèces peu chitinisées.

\section{EsPèces RENCONTRIÉES}

A. Pterolichinæ. - Dans cette sous-famille les pattes antérieures sont inermes. Les femelles ont l'abdomen entier et sont pourvues d'une plaque chitineuse notogastrique. Treize espèces ont été identifiées par nous au Maroc appartenant à cette sous-famille.

Genre Falculifer. - Caractérisé par le grand développement des pattes antéri sures chez les mâles hétéromorphes (Trouessart, 1886). Ce genre est inféodé aux columbiformes.

Falculifer rostratus Buchholz a été récolté sur Streptopelia turtur arenicola et chez Columba palumbus. Ce parasite est fréquent au Maroc shez le pigeon domestique. 
Genre Freyana. - Caractérisé par un corps orbiculaire et l'insertion infère des pattes postérieures.

$F$. anatina Koch, parasite fréquent des anatides domestiques ou sauvages, a été trouvé sur Anas crecca.

$F$. pelargica Megnin et Trouessart, parasite de diverses cicognes de l'ancien monde, a été retrouvé sur Ciconia ciconia.

Genre Pterolichus. - Cinq espèces du genre type de la sous-famille ont été identifiées.

$P$. charadrii Canestrini, sur Charadrius dubius.

$P$. delibatus Robin et Mégnin, sur Corvus corax tingitanus.

$P$. securiger Robin et Mégnin, sur Apus pallidus.

$P$. vanelli Canestrini, sur Vanellus vanellus.

$P$. xiphiurus, sur Glareola pratincola.

Chacune de ces espèces se trouve ici chez un hôte classique ou très voisin de l'hôte normal.

Genre Syringobia. - Corps allongé, sillon thoracique très profondément marqué. Les animaux appartenant à ce genre vivent non sur les plumes, mais dans les tuyaux creux de celles-ci, rémiges surtout.

S. tricalcarata Trouessart a été trouvé sur Charadrius dubius.

Genre Xoloptes. - Caractérisé par un développement anormal de la $\mathrm{VI}^{\circ}$ paire de pattes chez le mâle.

$X$. claudicans Robin et Megnin a été récolté sur Alectoris barbara.

Genre Pteronyssus. - Les mâles présentent un développement remarquable de la $\mathrm{III}^{\circ}$ paire de pattes, ce qui les rapproche des espèces appartenant à la sous-famille suivante, dans laquelle le genre Pteronyssus a été classé parfois. Mais les pattes antérieures sont inermes chez les Pteronyssus, alors qu'elles sont armées chez les Analgesinx. Trois espèces ont été identifiées :

$P$. infuscatus Trouessart, chez Hirundo rustica.

$P$. nuntiaveris Berlèse, chez le même hôte (Pl. II $c$ ).

P. striatus Robin, chez Fringilla spodiogenys.

Ces hôtes sont classiques pour les trois espèces.

B. Analgesinæ. - Cette sous-famille se caractérise par la présence de mâles hétéromorphes, différant des femelles par le grand développement d'une ou de deux paires de pattes postérieures. Les femelles ont l'abdomen entier et sont généralement dépourvues de plaque notogastrique. Les pattes antérieures sont armées dans les 
deux sexes. Nous avons identifié neuf espèces appartenant à cette sous-famille.

Genre Analges. - La troisième paire de pattes du mâle, très hypertrophiée, ne porte pas d'ambulacre.

A. chelopus Hermann a été trouvé régulièrement sur Passer domesticus tingitanus. Seuls, trois moineaux capturés en hiver dans le Maroc présaharien n'étaient pas parasités. Le moineau est hôte normal pour cet acarien que nous avons aussi rencontré, mais très accidentellement, sur le chardonneret et sur le cini.

Sur Sylvia atracapilla, nous avons régulièrement trouvé une variété d'A. chelopus, véritable caricature du type. La troisième paire de pattes est encore plus largement hypertrophiée. Son troisième article présente une large encoche à la partie antérieure du bord externe et les dents du bord interne sont longues et très espacées l'une de l'autre. Le tubercule latéral qui se devine sur le flane d'A. chelopus, en avant de l'insertion de la troisième paire de pattes, se transforme chez la variété en un long couteau atteignant l'extrémité supérieure du deuxième article de la troisième patte.

La révision des espèces européennes voisines ou synonymes d'A. chelopus (A. clavipes, A. coleopteroides, A. fringillarum, A. incertus, A. nitzschii, A. spiniger) étant encore à faire (Trouessart, 1886), nous nous bornons à donner une photographie du mâle hétéromorphe de la variété (PI. II $a$ ) et une du mâle hétéromorphe du type (Pl. II $b$ ) pour illustrer les différences.

C'est encore à une variété d'A. chelopus, qu'il faut sans doute rapporter quelques acariens trouvés chez un Phylloscopus sp.

A. (Analgopsis) passerinus (L), parasite des fringillidés, a été trouvé chez Carduelis carduelis, Carduelis cannabina, Fringilla s/:odiogenys africana, Serinus canaria (PI. I). Nous l'avons aussi observé chez un turdidé : Saxicola torquata.

C'est encore à $A$. passerinus que nous rapportons de rares Analges récoltés sur Anthus trivialis (et non à $A$. tridentatus, décrit des alaudidés et retrouvé par nous sur Anthus richardi, en Indochine).

C'est en revanche à $A$. tridentatus, que nous rapportons quelques acariens récoltés sur Sturnus vulgaris.

Genre Megninia. - La troisième paire de pattes du mâle, très hypertrophiée, porte un ambulacre. Le tarse de la quatrième patte est normal.

Quatre espèces ont été identifiées :

M. xstivalis Berlèse, chez Apus pallidus brehmorum. 
ANNALES DE PARASITOLOGIE

T. XXIII, Nos 1·2, 1948.
Planche I

(Mémoire Gaud el Petitot)

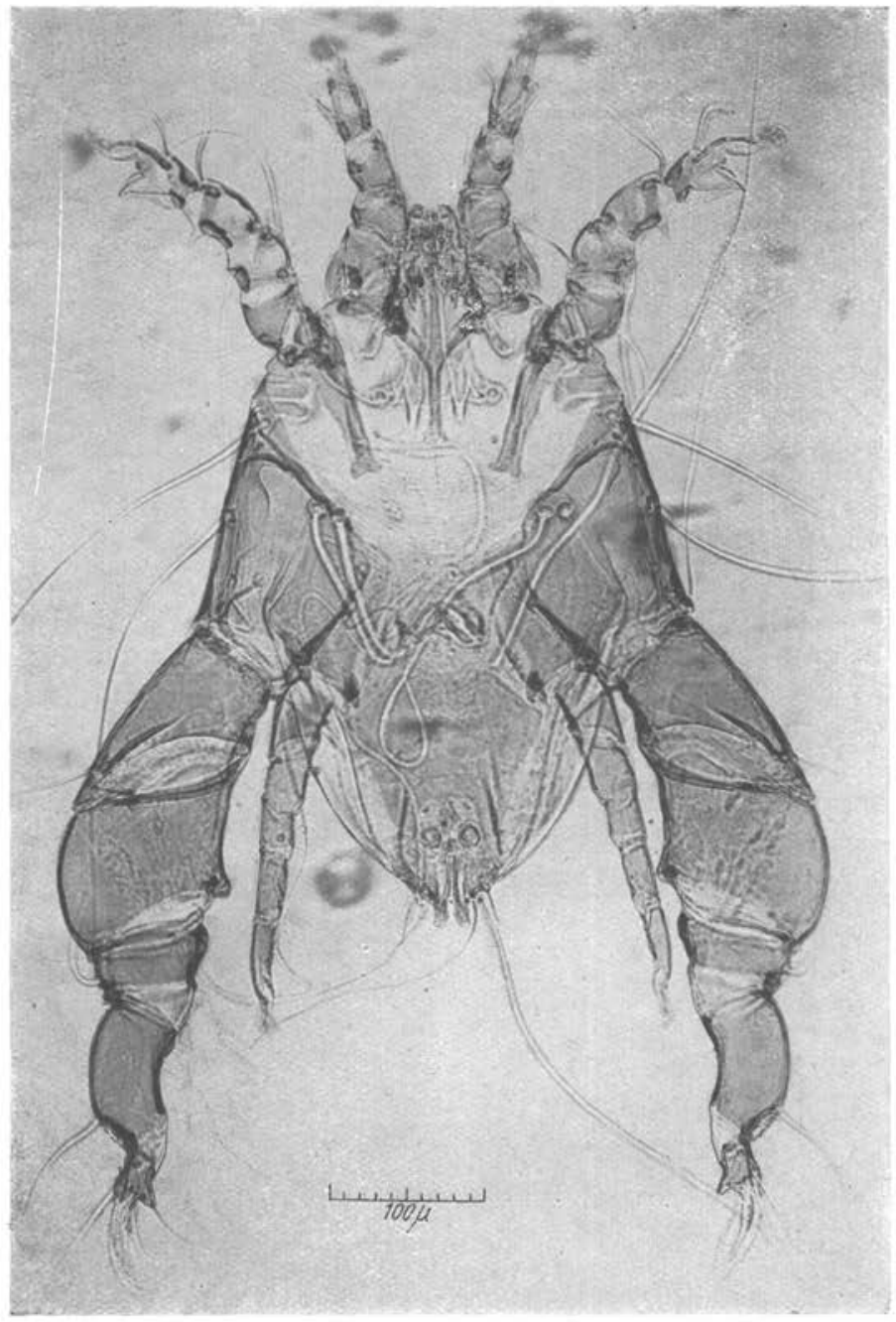

Planche 1

shalges (Analgopsis) passerinus (L.). 


\section{P'LANCHe II}

a. Analges chelopus Hermann. - Mâle hétéromorphe de la variété.

b. Analges chelopus Hermann. - Mâle hétéromorphe du type.

c. Pteronyssus nuntiæveris Berlèse.

d. Trouessartia minutipes Berlèse. 

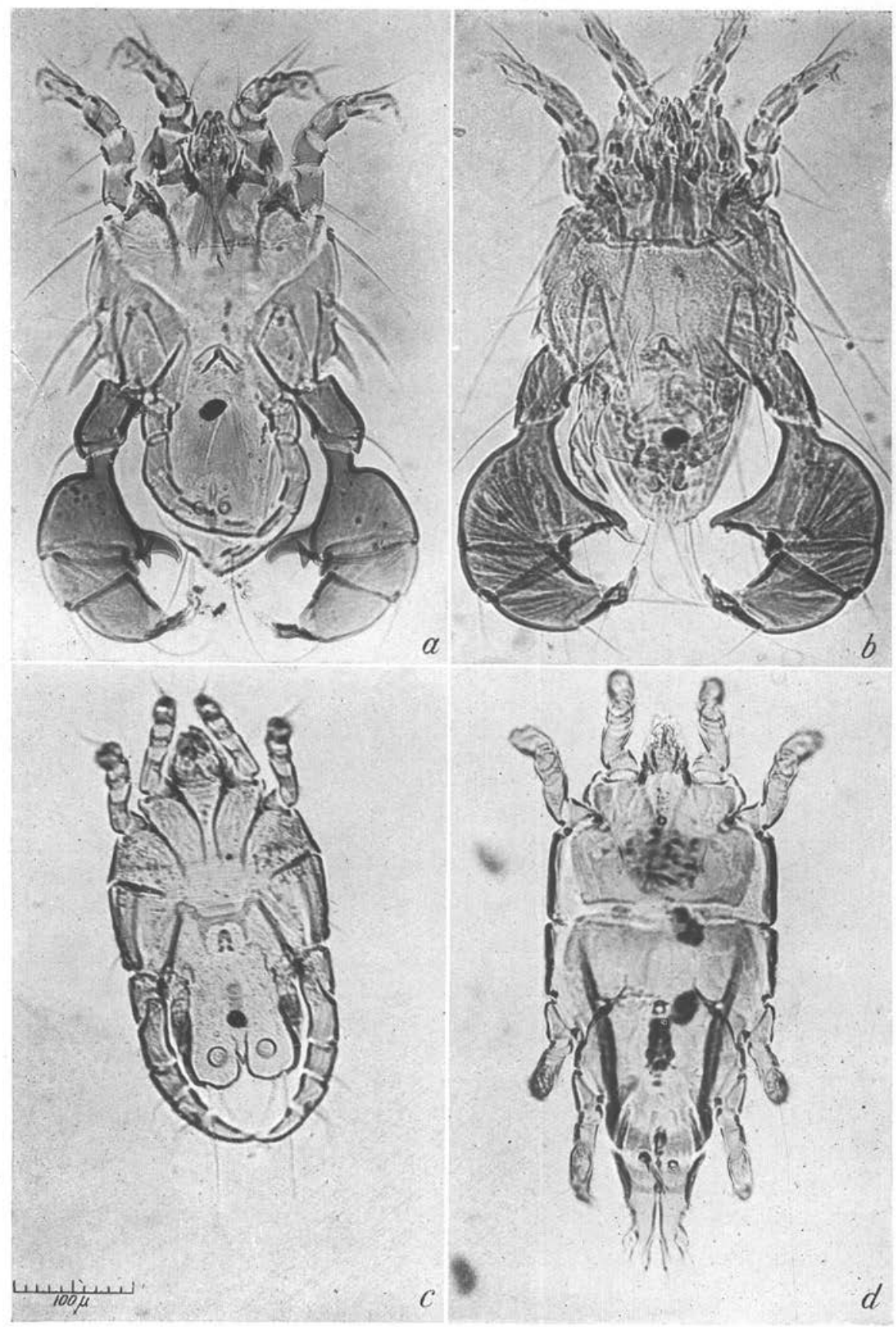

M. centropoda Mégnin, chez Vanellus vanellus.

M. columbre Buchholz, chez Columba livia, Columba palumbus, Streptopelia turtur arenicola.

Nous avons récolté aussi cette dernière espèce chez une Sylvia simplex trouvée morte depuis peu. $M$. columbre, trop abondant pour y être accidentel, y voisinait avec Pterophagus strictus, autre parasite normal des columbiformes.

M. tetraonis Trouessart a été trouvé sur Alectoris barbara. Chez Parus cæruleus enfin, nous avons trouvé deux exemplaires d'une Megninia voisine de M. megacentros (fig. 1, A et B).

A

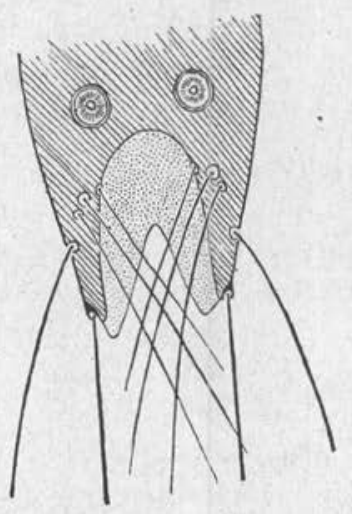

B

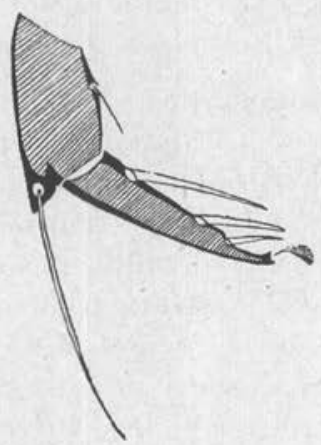

Fig. 1. - A. Extrémité abdominale dư mâle chez Megninia de Parus caruleus. is. Extrémité d'une patte de la $3^{\text {e }}$ paire chez le même.

Genre Mesalges. - Comme Megninia, mais le tarse de la quatrième paire de pattes du mâle atrophié. Les femelles ont souvent une plaque notogastrique.

Une seule et même espèce a été rencontrée chez divers fringillidés. Chloris chloris, Fringilla spodiogenus, Carduelis carduelis, Carduelis cannabina, Serinus canaria. Il est difficile d'affirmer s'il s'agit de $M$. oscinum Trouessart, ou de $M$. similis Bonnet. A s'en tenir à la description de Bonnet (1924), la forme des lobes abdominaux du mâle est celle de $M$. similis. La taille, cependant, est plutôt celle de $M$. oscinum. Ces deux espèces semblent d'ailleurs extrêmement voisines.

Genre Protalges. - Les deux paires de pattes postérieures sont toutes deux également hypertrophiées.

De ce genre, considéré comme la forme la plus ancienne de la sous-famille, nous avons identifié deux espèces : 
P. accipitrinus Trouessart, chez Falco naumani.

$P$. attenuatus Trouessart, chez Tyto alba.

Ces hôtes sont normaux pour ces deux espèces.

c. Proctophyllodinæ. - Dans cette sous-famille, à peu près inféodée aux petits passereaux, l'abdomen est bilobé dans les deux sexes et porte généralement des ornements chitineux divers (poils gladiformes, feuilles transparentes, etc...).

Douze espèces ont été identifiées.

Genre Alloptes. - La IV ${ }^{\circ}$ paire de pattes est hypertrophiée chez le mâle.

A. (Joubertia) microphyllus Robin et Mégnin a été trouvé régulièrement chez Fringilla spodiogenys africana et aussi chez Saxicola torquata. Le pinson est l'hôte habituel de cet acarien.

Genre Proctophyllodes. - Bonnet et Timon-David, examinant les sarcoptidés des oiseaux de Provence, rapportent à $P$. ampelidis Buchholz les Proctophyllodes trouvés chez nombre d'espèces de passereaux notamment: Anthus trivialis et sylvia atracapilla $(3 c)$.

Entrainés par leur autorité, nous avons été tentés de rapporter à $P$. ampelidis la majeure part de nos récoltes. L'aspect général des Proctophyllodes recueillis ne démentait pas cette façon de voir. Pourtant, un examen attentif des organes génitaux annexes mâles nous a fait retrouver des différences constantes que schématise la figure 2.

A la lumière de ces constatations, nous pensons devoir rapporter à $P$. ampelidis les acariens rencontrés habituellement chez :

Carduelis carduelis, Carduelis cannabina, Chloris chloris, Fringilla spodiogenys, Passer domesticus, Serinus canaria, et, accidentellement, chez :

Sylvia atracapilla, Columba livia. Alectoris barbara.

Par contre, quatre espèces voisines, mais différentes de $P$. ampelidis et différentes entre elles, parasitent : Motacilla alba, Emberiza calandra, Sylvia atracapilla, Anthus trivialis.

Ici encore, en attendant une révision des espèces européennes du genre Proctophyllodes, nous nous bornerons à figurer les caractères de ces espèces sans nommer d'espèces nouvelles. Ce serait compliquer encore l'établissement délicat de la synonymie du groupe " $P$. ampelidis, $P$. profusus, $P$. truncatus".

En dehors de ce groupe nous avons récolté :

P. arcuaticaulis Trouessart, sur Erythacus rubecula et sur Saxicola torquata. 
P. stylifer Buchholz, sur Parus cæruleus ultramarinus.

Genre Pterodectes. - Arc génital chitineux de la femelle raccordé aux épimères des pattes postérieures.

Deux espèces identifiées :

Pt. bilobatus Robin et Mégnin, chez Galerida cristata et Galerida teklas.
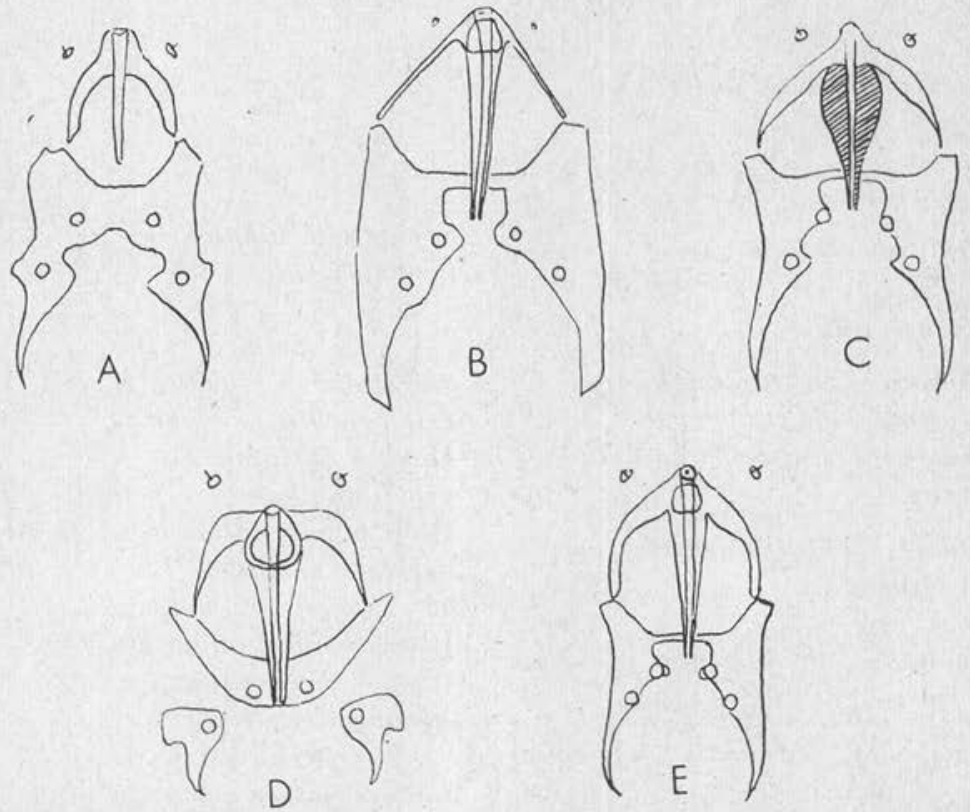

FIG. 2. - Organe génital et apodèmes chez les Proctophyllodes de quelques passereaux. - A, Motacilla alba. - B, Emberiza calandra. - C, Sylvia atracheilla. - D, Anthus trivialis. - E, Passer domesticus.

Pt. rutilus Robin et Mégnin, chez Turdus merula.

Genre Pterophagus. - Lobes abdominaux de la femelle élargis latéralement et sans appendices.

$P t$. strictus Robin et Mégnin, parasite habituel du pigeon domestique, a été récolté sur Sylvia simplex (voir plus haut, p. 41) en compagnie de Megninia columbæ.

Genre Trouessartia. - Lobes. abdominaux allongés et atténués dans les deux sexes, terminés chez le mâle par des appendices transparents en forme de nageoire de poisson ou de roue dentée. 
T. corvina Koch a été trouvé chez Sturnus vulgaris.

T. minutipes Berlèse a été récolté sur Hirundo rustica.

Chez Erythacus rubecula, nous avons recueilli quelques femelles et d'assez nombreuses nymphes de Trouessartia. En l'absence de mâles il nous est impossible de préciser l'espèce en cause.

\section{TABLEAU SELON LES HÔTES DES SARCOPTIDES PLUMiColes RENCONTRÉS AU MAROC}

\section{ACCIPITRIFORMES.}

Falco naumani Fleisch.

\section{STRYGIFORMES.}

Tyto alba Scopoli.

\section{PASSERTFORMES.}

Corvus corax tingitanus Irby.

Sturnus vulgaris L.

Passer domesticus tingitanus Loche.

Chloris chloris aurantiventris (Cabanis).

Fringilla spodiogenys africana Lev.

Carduelis carduelis africanus (Hartert).

Carduelis cannobina (L.).

Serinus canaria serinus (L.).

Emberiza calandra.

Galerida cristata riggenbachi Hartert et

Galerida tekla erlangeri Hartert.

Anthus trivialis L.

Motacilla alba L.

Turdus merula mauritanica (Hartert).

Erithacus rubecula L.

Saxicola torquata desfontainesi Blanchet.
Protalges accipitrinus.

Kramerella lunulata.

Protalges attenuatus.

Plerolichus delibatus.

Trouessartia corvina.

Analges chelopus.

Proctophyllodes ampelidis.

Alloptes microphyllus (accidentel ?)

Mesalges oscinum.

Prcctophyllodes ampelidis.

Alloptes microphyllus.

Analges passerinus.

Pteronyssus striatus.

Analges passerinus.

Mesalges oscinum.

Prcctophyllodes ampelidis.

Proctophyllodes sp.?

Pterodectes bilobatus.

Proctophyllodes sp.?

Analges passerinus.

Proctophyllodes sp.?

Proctophyllodes sp.?

Pterodectes rutilus.

Proctophyllodes arcuaticaulis.

Trouessartia sp.?

Analges passerinus.

Proctophyllodes arcuaticaulis. 
TABLeaU SElon les hôtes des SARCoptides PLumicoles RENCONTRÉS AU MAROC

(suite)

Sylvia atracapilla L.

Analges chelopus (variété ?).

Proctophyllodes ampelidis.

Proctophyllodes sp.?

Sylvia communis Lathan.

Megninia columba.

Pterophagus strictus (accidentels).

Phylloscopus s.p.?

Analges chelopus (variété ?).

Proctophyllodes arcuaticaulis.

Parus caruleus ultramarinus Bonaparte.

Megninia sp.? voisine de megacentros.

Proctophyllodes stylifer.

Hirundo rustica L.

Pteronyssus infuscatus.

Pteronyssus nuntiaveris.

Trouessartia minutipes.

MiCROODIFORMES.

Apus pallidus brehmorum Har- Megninia astivalis. tert.

Pterolichus securiger.

Columbiformes.

Columba palumbus excelsus Bonaparte.

Columba livia Gmelin.

Columba livia Gmelin.
$\begin{gathered}\text { Streptopelia turtur } \\ \text { (Hartert). }\end{gathered}$$\left\{\begin{array}{l}\text { Falculifer rostratus. } \\ \text { Megninia columba. }\end{array}\right.$

Galliformes.

Alectoris barbara (Bonnaterre).

\section{Charadriformes.}

Glareola pratincola.

Charadrius dubius curonicus Gmelin.

Vanellus vanellus (Linne).

Scolopax rusticola.

Megninia tetraonis.

Proctophyllodes ampelidis (accidentel).

Xoloptes claudicans.

Pterolichus xyphiurus.

Pterolichus charadrii.

Syringobia tricalcarata.

Megninia centropoda.

Pterolichus vanelli.

Proctophyllodes sp.?

ArdeIformes.

Ciconia ciconia L.

Freyana pelargica.

Axatiformes.

Anas crecca $\mathrm{L}$.

Freyana anatina. 


\section{BIBLIOGRAPHIE}

ANdrÉ (M.). - Sur les Sarcoptides plumicoles des Ratitx. Bull. du Muséum, $2^{\circ}$ s., X, 1938, p. 590.

Bonvet (A.). - Révision des genres Megninia, Mesalges et genres voisins de la sous-famille des Sarcoptidés plumicoles. Bull. Soc. Zool. de France, XLIX, 1924, p. 190.

Bonnet (A.) et Timon-David (J.), - Contribution à l'étude des acariens plumicoles. a) $1^{\text {re }}$ note, Bull. Soc. Linnéenne de Pruvence, V, 1932 ; b) $2^{\circ}$ note, Ann. Parasit. hum. et comp., XI, 1933, p. 442 ; c) $3^{e}$ note, Ibid., XII, 1934, p. 257.

MÉgnin (P.). - Sur les métamorphoses des acariens de la famille des Sarcoptidés et de celle des Gamasidés. C.R. Acad. Sc., LXXVIII, 1874.

Trouessart. - Diagnoses d'especes nouvelies de sarcoptidés plumicoles.

Bull. Soc. Et. Sc. Angers, XVI, 1886, p. 92 .
Révision des genres de la sous-famille des Analgesina ou Sarcoptidés plumicoles. Bull. Soc. Zool. de France, XL, 1915, p. 207.

Instilut a'hygiène de Rabal (Maroc) 